\title{
Inserción de litio en varios molibdatos preparados por dos métodos de síntesis
}

\author{
R. HERRERA SÁNCHEZ; A. F. FUENTES*; A. MARTíNEZ-DE LA CRUZ AND L. M. TORRES-MARTíNEZ \\ Facultad de Ciencias Químicas, División de Estudios Superiores, Universidad Autónoma de Nuevo León, Monterrey, Nuevo León, México
}

En este trabajo se presenta un estudio de la inserción electroquímica de litio en molibdatos de fórmula general $\mathrm{MMoO}_{4}(\mathrm{M}=$ $\mathrm{Zn}, \mathrm{Ni}, \mathrm{Mn}, \mathrm{Cu}$ y $\mathrm{Co}$ ) y en $\mathrm{Al}_{2}\left(\mathrm{MoO}_{4}\right)_{3}$ preparados por reacción de estado sólido (RES) y por reacción en solución (RS). El estudio electroquímico mostró que en general, no existen diferencias fundamentales en el comportamiento de los molibdatos sintetizados por uno u otro método y con semejante grado de cristalinidad. Sin embargo, los compuestos preparados por RS y calcinados a temperaturas intermedias incorporaron una mayor cantidad de litio que los obtenidos por RES aunque en todos los casos se observó una transformación irreversible de los compuestos originales con pérdida casi completa de la cristalinidad durante la primera descarga de la celda. La cantidad de átomos de litio incorporados en estos compuestos fue muy elevada $(\mathrm{Li} / \Sigma \mathrm{M} \approx 4)$ aunque de forma irreversible.

Plabras clave: Insercción de litio, molibdatos

Lithium insertion in several molybdates prepared by two synthetic routes

In this work we present a study we present a comparative study of lithium insertion in several compounds of general formula $\mathrm{MMoO}_{4}(\mathrm{M}=\mathrm{Zn}, \mathrm{Ni}, \mathrm{Mn}, \mathrm{Cu}, \mathrm{Co})$ and in $\mathrm{Al}_{2}\left(\mathrm{MoO}_{4}\right)_{3}$, prepared by solid state reaction (RES) and by solution and precipitation (RS). The electrochemical study showed no difference on the performance of the compounds prepared by these two methods and with a similar degree of crystallinity. However, those compounds prepared by RS and fired to intermediate temperatures, incorporated a larger amount of lithium atoms although in all cases an irreversible transformation of the pristine materials was observed with a complete loss of crystallinity after the initial discharge. The amount of lithium atoms incorporated was large although the insertion is not reversible and most of them are not extracted afeter completing a charge-discharge cycle.

\section{Keywords: lithium insertion, molybdat}

\section{INTRODUCCIÓN}

La búsqueda de nuevos materiales para su aplicación en sistemas de almacenamiento de energía se ha constituído como una de las áreas de la ciencia de materiales de mayor actualidad. Los diferentes grupos de investigación que trabajan en este campo centran su actividad en dos direcciones: (i) por un lado tratan de buscar nuevos materiales susceptibles de ser usados como electrodo positivo en estos dispositivos o mejorar las propiedades de los óxidos cerámicos ya en uso de sistemas comercializados $\left(\mathrm{LiCoO}_{2}, \mathrm{LiNiO}_{2}\right.$ y $\left.\mathrm{LiMn}_{2} \mathrm{O}_{4}\right)$ y (ii) por el otro buscan alternativas a los electrodos de carbón actualmente en uso. Recientemente, diferentes autores (1-2) propusieron vanadatos de litio o de metales de transición como posibles electrodos en dispositivos de almacenamiento de energía. Estos compuestos, ya sea cristalinos y descargados hasta potenciales lo suficientemente bajos como para volverlos amorfos o sintetizados por química suave y usados directamente amorfos, presentan características electroquímicas muy interesantes y capacidades del orden de 800 ó $900 \mathrm{Ah} / \mathrm{Kg}$, valores bastante superiores a los que se obtienen con los sistemas actualmente en uso. Leroux et al. (3) evaluaron el comportamiento del $\mathrm{Na}_{\mathrm{x}} \mathrm{MoO}_{3}(\mathrm{x} \approx 0,25)$ en celdas electroquímicas descargadas a potenciales muy bajos observando que la inserción de litio en este compuesto es reversible y obteniendo capacidades del orden de $600 \mathrm{Ah} / \mathrm{kg}$. A la luz de todos estos trabajos y de los resultados obtenidos por nosotros mismos con otro óxido de molibdeno (4), nos planteamos la conveniencia de realizar un estudio de la inserción de litio en diferentes molibdatos de metales representativos, preparados por dos métodos diferentes de síntesis.

\section{EXPERIMENTAL}

\subsection{Síntesis}

La preparación de los compuestos objetos de este estudio se realizó por dos métodos diferentes de síntesis: reacción en estado sólido (RES) y reacción en solución (RS). Para la síntesis por RES se partió de mezclas de $\mathrm{MoO}_{3}$ y del óxido del metal correspondiente en la proporción estequiométrica adecuada $\left(\mathrm{MoO}_{3}: \mathrm{MO}\right.$ o $\left.\mathrm{Al}_{2} \mathrm{O}_{3}\right)$. Estas mezclas se colocaron en ampollas de cuarzo que fueron selladas para prevenir pérdidas por volatilización del $\mathrm{MoO}_{3}$ y sometidas a tratamiento térmico a temperaturas comprendidas entre $550-1100^{\circ} \mathrm{C}$ según el compuesto a obtener. El $\mathrm{MoO}_{3}$ de partida se obtuvo calentando molibdeno metálico durante 5 horas a $500^{\circ} \mathrm{C}$ en un horno eléctrico.

La síntesis por RS se llevó a cabo mezclando volúmenes en relación estequiométrica de una solución 0,05 $\mathrm{M}$ de molibdato amónico $\left[\left(\mathrm{NH}_{4}\right)_{8} \mathrm{Mo}_{10} \mathrm{O}_{34}\right]$ y de soluciones $0,25 \mathrm{M}$ de los nitra- 
tos hidratados de níquel, cobalto, cobre, manganeso, zinc y aluminio. En algunos casos se observó la aparición instantánea de cierta cantidad de precipitado al mezclar las dos soluciones por los que para asegurar una reacción completa y homogénea, el precipitado fue redisuelto añadiendo $\mathrm{HNO}_{3} 3 \mathrm{~N}$ hasta $\mathrm{pH}$ 1. La precipitación de los productos de reacción se consiguió mediante la adición de $\mathrm{NH}_{4} \mathrm{OH} 3 \mathrm{~N}$ y calentamiento a $50^{\circ} \mathrm{C}$ en un baño de agua. El precipitado se filtró, lavó varias veces con agua desionizada y secó a $85^{\circ} \mathrm{C}$ durante 12 horas.

La caracterización de los productos de reacción se llevó a cabo mediante difracción de rayos- $X$ en polvos usando un equipo SIEMENS D-5000 con radiación CuK $\alpha$. El análisis térmogravimétrico (ATG) y térmico diferencial (ATD) se llevó a cabo en un equipo TA Instruments SDT 2960 usando pesos aproximados de muestra de $15 \mathrm{mg}$ y velocidades de calentamiento de $10^{\circ} \mathrm{C} / \mathrm{min}$.

\subsection{Estudio Electroquímico}

El estudio electroquímico se llevó a cabo en un sistema multicanal potenciostato-galvanostato MacPile II (5) usando celdas tipo Swagelok ${ }^{\mathrm{TM}}$ y configuradas de la siguiente forma: $\mathrm{Li} /$ electrolito/ $\mathrm{MMoO}_{4}$. Se usaron dos electrolitos: (i) solución $1 \mathrm{M}$ de $\mathrm{LiClO}_{4}$ en una mezcla 1:1 de carbonato de etileno (EC) y dietoxietano (DEE) y (ii) solución $1 \mathrm{M}$ de $\mathrm{LiClO}_{4}$ en una mezcla 2:1 de carbonato de etileno (EC) y dimetil carbonato (DMC). El cátodo se preparó mezclando la fase en estudio con carbón amorfo y con un aglutinante orgánico (etilen-propilendien-terpolímero al 0,5\% p/v en ciclohexano) en la proporción 89:10:1 (\% en peso). El ensamblaje de la celda se llevó a cabo en una caja seca en atmósfera de argón. El estudio electroquímico se llevó a cabo mediante experimentos en modo galvanostático o en modo potenciostático (SPECS) en los intervalos de potencial de 3 a $0,5 \mathrm{~V}$ y de 3 a $0,02 \mathrm{~V}$ vs. $\mathrm{Li}^{+} / \mathrm{Li}$. La toma de rayos- $X$ in situ se llevó a cabo usando una celda electroquímica especialmente diseñada para poder ser acoplada al difractómetro SIEMENS-D-5000.[4]
TABLA 1. CONDICIONES EXPERIMENTALES PARA LA SÍNTESIS DE $\mathrm{MMOO}_{4} \mathrm{y}$ $\mathrm{Al}_{2}\left(\mathrm{MoO}_{4}\right)_{3}$

\begin{tabular}{|l|c|c|}
\hline COMPUESTO & $\begin{array}{c}\text { TEMPERATURA } \\
\left({ }^{\circ} \mathbf{C}\right)\end{array}$ & $\begin{array}{c}\text { TIEMPO } \\
\text { (horas) }\end{array}$ \\
\hline $\mathrm{MnMoO}_{4}$ & 900 & 24 \\
\hline $\mathrm{ZnMoO}_{4}$ & 550 & 24 \\
\hline $\mathrm{NiMoO}_{4}$ & 1100 & 48 \\
\hline $\mathrm{CoMoO}_{4}$ & 900 & 24 \\
\hline $\mathrm{CuMoO}_{4}$ & 650 & 24 \\
\hline $\mathrm{Al}_{2}\left(\mathrm{MoO}_{4}\right)_{3}$ & 850 & 30 \\
\hline
\end{tabular}

\section{RESULTADOS}

\subsection{Síntesis por Reacción de Estado Sólido}

La síntesis de los molibdatos en estudio por estado sólido rindió siempre productos puros no observándose por difracción de rayos-X en polvos, la presencia de ninguna otra fase. La tabla I resume los parámetros experimentales utilizados en la síntesis de estos compuestos.

\subsection{Síntesis por Reacción en Solución}

La síntesis por reacción en solución de los diferentes compuestos en estudio rindió los resultados que se resumen en la tabla 2 y que se explican detalladamente a continuación.

\section{MOLIBDATO DE COBRE}

Al mezclar las soluciones de $\left(\mathrm{NH}_{4}\right)_{8} \mathrm{Mo}_{10} \mathrm{O}_{34} \mathrm{y}$ $\mathrm{Cu}\left(\mathrm{NO}_{3}\right)_{2} \cdot 2.5 \mathrm{H}_{2} \mathrm{O}$ se obtuvo una solución de color azul claro y

TABLA 2. RESULTADOS OBTENIDOS EN LA SÍNTESIS DE LOS DIFERENTES MOLIBDATOS POR REACCIÓN EN SOLUCIÓN

\begin{tabular}{|c|c|c|c|c|}
\hline $\begin{array}{l}\text { Compuesto } \\
\text { buscado }\end{array}$ & $\begin{array}{c}\text { Precipitado } \\
\text { obtenido }\end{array}$ & PH & $\begin{array}{c}\mathrm{T}\left({ }^{\circ} \mathrm{C}\right) \\
\text { calcinación }\end{array}$ & Observaciones \\
\hline $\mathrm{CoMoO}_{4}$ & $\mathrm{Co}_{1,2} \mathrm{MoO}_{4,2 \cdot 1,3} \mathrm{H}_{2} \mathrm{O}$ & 7 & 950 & $\begin{array}{c}\text { La solución inicial presentó color rojo } \\
\text { y pH 4,4. Precipitados de color } \\
\text { rosa y violeta aparecieron desde } \\
\text { pH } 6 \text { a pH } 9 \text { todos a temperatura ambiente }\end{array}$ \\
\hline $\mathrm{CuMoO}_{4}$ & $\left(\mathrm{NH}_{4}\right)_{2} \mathrm{Cu}\left(\mathrm{MoO}_{4}\right)_{2}$ & 6 & 750 & $\begin{array}{c}\text { Solución de color azul claro y pH } 6 \text {. } \\
\text { Desde } \mathrm{pH} 4 \text { a pH } 9 \text { se obtuvieron precipitados } \\
\text { de color verde claro }\end{array}$ \\
\hline $\mathrm{MnMoO}_{4}$ & $\begin{array}{c}\mathrm{MnMoO}_{4} \cdot \mathrm{H}_{2} \mathrm{O} y \\
\left(\mathrm{NH}_{4}\right)_{6} \mathrm{Mo}_{7} \mathrm{O}_{24} \cdot 4 \mathrm{H}_{2} \mathrm{O}\end{array}$ & 7 & 900 & $\begin{array}{l}\text { Solución inicial de color amarillo. } \\
\text { Se obtuvo precipitado de color amarillo } \\
\text { pálido desde } \mathrm{pH} 4 \text { a pH } 9\end{array}$ \\
\hline $\mathrm{NiMoO}_{4}$ & $\mathrm{NiMoO}_{4} \cdot \mathrm{H}_{2} \mathrm{O}$ & 6,5 & 900 & $\begin{array}{l}\text { Solución inicial de color verde } \\
\text { y pH 4,5. Para obtener precipitado fue necesario } \\
\text { elevar el pH hasta } 6,5 \text { y calentar a } 70^{\circ} \mathrm{C} \text {. }\end{array}$ \\
\hline $\mathrm{ZnMoO}_{4}$ & $\mathrm{Zn}_{5} \mathrm{Mo}_{2} \mathrm{O}_{11} \cdot 5 \mathrm{H}_{2} \mathrm{O}$ & 7 & 600 & $\begin{array}{l}\text { Solución inicial incolora y pH 4,6. } \\
\text { Precipitados a temperatura ambiente } \\
\text { desde } \mathrm{pH} 5 \text { a pH } 9\end{array}$ \\
\hline $\mathrm{Al}_{2}\left(\mathrm{MoO}_{4}\right)_{3}$ & Fase amorfa & 7 & 700 & $\begin{array}{l}\text { Solución inicial de color amarillo y pH 1,3. } \\
\text { Precipitados de color blanco se obtuvieron } \\
\text { desde } \mathrm{pH} 4 \text { hasta } \mathrm{pH} 8\end{array}$ \\
\hline
\end{tabular}


TABLA 3. NÚMERO DE ÁTOMOS DE LITIO INCORPORADOS POR ÁTOMO METÁLICO $(\mathrm{Li} / \Sigma \mathrm{M} \approx 4)$ PARA LOS DIFERENTES COMPUESTOS ESTUDIADOS

\begin{tabular}{|c|c|c|c|}
\hline COMPUESTO & RES & $\begin{array}{c}\text { RS } \\
\text { (Menor T) }\end{array}$ & $\begin{array}{c}\text { RS } \\
\text { (Mayor T) }\end{array}$ \\
\hline $\mathrm{MnMoO}_{4}$ & 5 & 4 & 4.5 \\
\hline $\mathrm{ZnMoO}_{4}$ & 3.7 & - & - \\
\hline $\mathrm{NiMoO}_{4}$ & 3.7 & - & - \\
\hline $\mathrm{CoMoO}_{4}$ & 4.4 & 5.5 & 4.3 \\
\hline $\mathrm{CuMoO}_{4}$ & 4.7 & 7 & 4.7 \\
\hline $\mathrm{Al}_{2}\left(\mathrm{MoO}_{4}\right)_{3}$ & 3.6 & - & 3.6 \\
\hline
\end{tabular}

pH 6. Dicha solución fue sometida al tratamiento especificado anteriormente observándose la formación de un precipitado de color verde claro para valores de $\mathrm{pH}$ comprendidos entre 4 y 9. Este producto fue analizado por difracción de rayos- $X$ en polvos encontrándose que su difractograma era similar al que se describe en la literatura para el compuesto de fórmula molecular $\left(\mathrm{NH}_{4}\right)_{2} \mathrm{Cu}\left(\mathrm{MoO}_{4}\right)_{2}$ _[JCPDS 40-1490]. A continuación se procedió a someter el precipitado a tratamiento térmico a diferentes temperaturas mostrándose en la Fig.1, las transformaciones observadas en el difractograma del mismo. A $400^{\circ} \mathrm{C}$, el precipitado se descompuso produciendo una mezcla de dos molibdatos de cobre (II): $\mathrm{CuMoO}_{4}$ y $\mathrm{Cu}_{3} \mathrm{Mo}_{2} \mathrm{O}_{9}$. Un posterior tratamiento térmico a $750^{\circ} \mathrm{C}$ dio como producto mayoritario $\mathrm{CuMoO}_{4}$. El estudio realizado por ATD-ATG reveló la existencia hasta $800^{\circ} \mathrm{C}$ de un solo pico exotérmico $\left(\approx 300^{\circ} \mathrm{C}\right)$ con una pérdida del $13 \%$ en peso hasta esa temperatura que debe corresponder al amonio contenido en el precipitado y al agua de hidratación. Debido a que nunca se pudo obtener por este método el $\mathrm{CuMoO}_{4}$ puro, solamente se estudió el comportamiento electroquímico del compuesto de cobre preparado por RES.

\section{MOLIBDATO DE COBALTO}

En este caso se partió de una solución de $\mathrm{Co}\left(\mathrm{NO}_{3}\right)_{2} \cdot 6 \mathrm{H}_{2} \mathrm{O}$ que al ser mezclada con la de molidato amónico produjo una solución de color rojo y pH 4,4. Se observó la aparición de precipitados de color rosa pálido a morado para valores de $\mathrm{pH}$ entre 6 y 9. Por difracción de rayos-X en polvos se identificó el precipitado como un compuesto de fórmula molecular $\mathrm{Co}_{1,3} \mathrm{MoO}_{4,3} \cdot 1,3 \mathrm{H}_{2} \mathrm{O}$ [JCPDS 14-0087]. Este precipitado se transformó a $400^{\circ} \mathrm{C}$ en el polimorfo de baja temperatura del $\mathrm{CoMoO}_{4}$ y a $950^{\circ} \mathrm{C}$ en el de alta temperatura sin ninguna otra fase presente.

\section{MOLIBDATO DE NÍQUEL}

La solución obtenida de mezclar el molibdato amónico con $\mathrm{Ni}\left(\mathrm{NO}_{3}\right)_{2} \cdot 6 \mathrm{H}_{2} \mathrm{O}$ resultó ser de color verde con un $\mathrm{pH} 4,5$ observándose la aparición de precipitado a $\mathrm{pH}$ 6,5. Aunque el difractograma del precipitado presentó algunas reflexiones, no fue posible su identificación química. Su tratamiento térmico a $400^{\circ} \mathrm{C}$ rindió un producto en el que ya eran visibles las principales reflexiones del $\mathrm{NiMoO}_{4}$ aumentando la cristalinidad del mismo a medida que se incrementaba la temperatura (Fig.2) sin ninguna otra fase presente identificada por difracción de rayos-X.

\section{MOLIBDATO DE ZINC}

En el caso del zinc se partió del nitrato tetrahidratado obteniéndose al añadir el molibdato amónico, una solución incolora de $\mathrm{pH} 4,6$ y observándose la aparición de precipitado para valores de $\mathrm{pH}$ comprendidos entre 5 y 9 . El precipitado cristalino obtenido fue identificado por difracción de rayos- $\mathrm{X}$ como el compuesto de fórmula molecular $\mathrm{Zn}_{5} \mathrm{Mo}_{2} \mathrm{O}_{11} \cdot 5 \mathrm{H}_{2} \mathrm{O}$ [JCPDS 30-1486]. Este compuesto tetragonal se transformó al calentar en $\mathrm{ZnMoO}_{4}$ único producto identificado en los patrones de difracción del precipitado calentado a 400 y $600^{\circ} \mathrm{C}$ y mostrados en la Fig.3. Se registraron las curvas ATD-ATG del precipitado obtenido de solución observándose un único evento exotérmi-

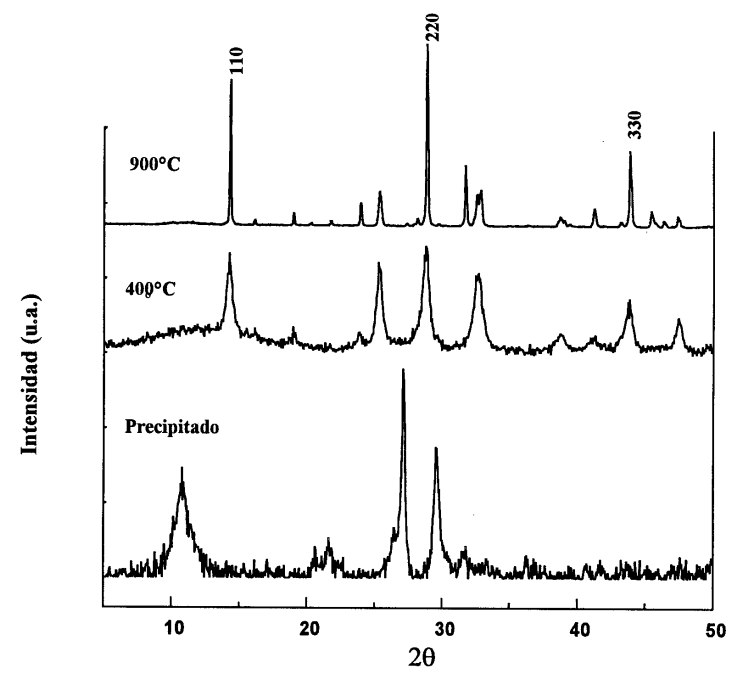

Figura 2.- Evolución del precipitado obtenido de la reacción del molibdato amónico y el nitrato hidratado de níquel con la temperatura.
Figura 1.- Evolución del precipitado obtenido de la reacción del molibdato amónico y el nitrato hidratado de cobre con la temperatura. 
Co a $259^{\circ} \mathrm{C}$ con una pérdida en peso asociada del 11,5\% Fig. 4 . Teniendo en cuenta que la pérdida de las 5 moléculas de agua del compuesto identificado por rayos- $X$ equivaldría exactamente a un $11,46 \%$ del peso original y que el único producto identificado por difracción de rayos- $X$ del precipitado calentado a temperaturas superiores a $300^{\circ} \mathrm{C}$ es el $\mathrm{ZnMoO}_{4^{\prime}}$ podemos sugerir que a $259^{\circ} \mathrm{C}$ se produce la descomposición del $\mathrm{Zn}_{5} \mathrm{Mo}_{2} \mathrm{O}_{11} \cdot 5 \mathrm{H}_{2} \mathrm{O}$. La descomposición de un mol de este compuesto podría originar a lo sumo dos moles de $\mathrm{ZnMoO}_{4}$ por lo que sería lógico pensar que existan también en el producto de su descomposición, tres moles de $\mathrm{ZnO}$ no identificadas por difracción de rayos- $X$ en polvos.

\section{MOLIBDATO DE MANGANESO}

La solución resultante de mezclar el molibdato amónico y el $\mathrm{Mn}\left(\mathrm{NO}_{3}\right)_{2}$ presentó una coloración amarilla obteniéndose precipitación para un valor de $\mathrm{pH}$ comprendido entre 5 y 9 . No se pudo identificar el precipitado por difracción de rayos-X en polvos aunque el tratamiento térmico del mismo produjo a temperaturas superiores $\mathrm{MnMoO}_{4}$, sin que se observase por esta técnica, la presencia de ningún otro compuesto.

\section{MOLIBDATO DE ALUMINIO}

Al mezclar las soluciones de molibdato amónico y $\mathrm{Al}\left(\mathrm{NO}_{3}\right)_{3} \cdot 9 \mathrm{H}_{2} \mathrm{O}$ se obtuvo una solución de color amarillo y $\mathrm{pH}$ 1.3. A valores de $\mathrm{pH}$ comprendidos entre 4 y 8 , se obtuvo un precipitado de color blanco con un difractograma típico de un compuesto amorfo con ausencia completa de reflexiones. Al tratar esta muestra a $700^{\circ} \mathrm{C}$ se obtuvo un producto cuyo patrón de difracción resultó ser idéntico al descrito en la literatura para el $\mathrm{Al}_{2}\left(\mathrm{MoO}_{4}\right)_{3}$.

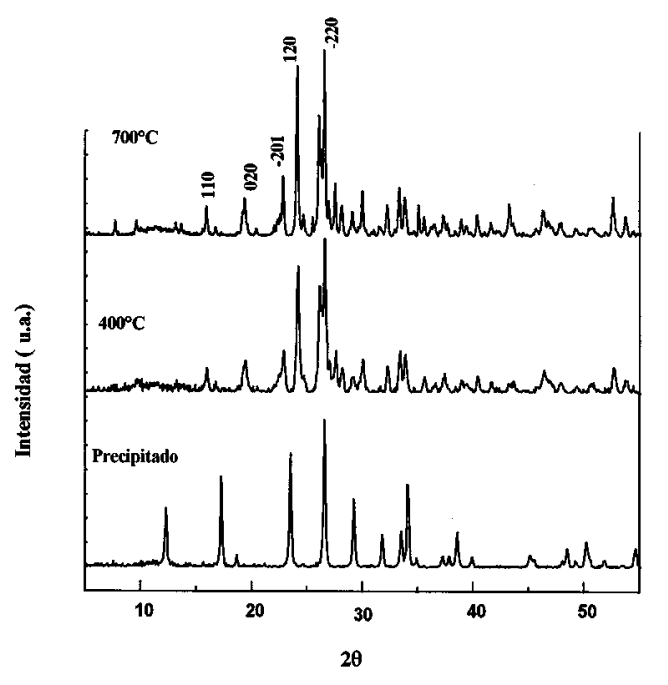

Figura 3.- Evolución del precipitado obtenido de la reacción del molibdato amónico y el nitrato hidratado de zinc con la temperatura.

\subsection{Estudio Electroquímico}

En la tabla 3 se resumen los resultados obtenidos en el estudio electroquímico de los 6 molibdatos estudiados y sintetizados por reacción de estado sólido y por reacción en solución.

Casi todas las curvas de voltaje vs. Composición, mostraron una caída inicial del voltaje de la celda hasta potenciales aproximados de $1.5 \mathrm{~V}$ seguida de cambios suaves de pendiente y una meseta de potencial aproximadamente constante alrededor de $0,5 \mathrm{~V}$ que probablemente se deba a la reducción de los metales presentes en los compuestos.

Una característica general que se observa en todas las celdas descargadas hasta $0,02 \mathrm{~V}$ es que el número de iones litio incorporados y por tanto de electrones, por fórmula (por encima de 8) sugiere una reducción completa de los dos metales presentes en los molidbatos $\left(\mathrm{Mo}^{6+} \mathrm{y} \mathrm{M}^{2+}\right)$ hasta su estado elemental. Solamente en el caso del molibdato de aluminio, el número de iones incorporados por fórmula (18) es menor a los que teóricamente harían falta para la reducción completa de los 3 átomos de $\mathrm{Mo}^{6+}$ y los dos de $\mathrm{Al}^{3+}$. En ningún caso fue posible determinar la presencia de estas especies en estado elemental por las técnicas de caracterización usadas en este trabajo si bien Leroux et al., en su trabajo con $\mathrm{Na}_{x} \mathrm{MoO}_{3}$ (3) y utilizando ${ }^{7} \mathrm{Li}$ RMN y EXAFS, mantienen que a bajo potencial se forma un material compuesto complejo y amorfo que consiste en un subóxido de Li-Mo en íntimo contacto con óxido de litio. Aunque la cantidad de litio incorporado es en general bastante elevada $(\mathrm{Li} / \Sigma \mathrm{M} \approx 4)$, la reacción de inserción es todos los compuestos estudiados irreversible ya que la mayor parte de los átomos inicialmente incorporados permanecen en la estructura aún después de completar un ciclo de carga-descarga. Otra característica común observada cuando las celdas en estudio se descargaron hasta $0,02 \mathrm{~V}$, es el completo deterioro de la estructura de partida durante la primera descarga resultando en curvas E vs. composición en las que cargas y descargas sucesivas presentan un perfil completamente diferente al de la primera descarga. La irreversibilidad de la reacción de inserción es independiente del método empleado para la síntesis de los materiales de partida ya que no se observaron cambios importantes en el comportamiento electroquímico de los compuestos estudiados preparados por uno u otro método. Sin

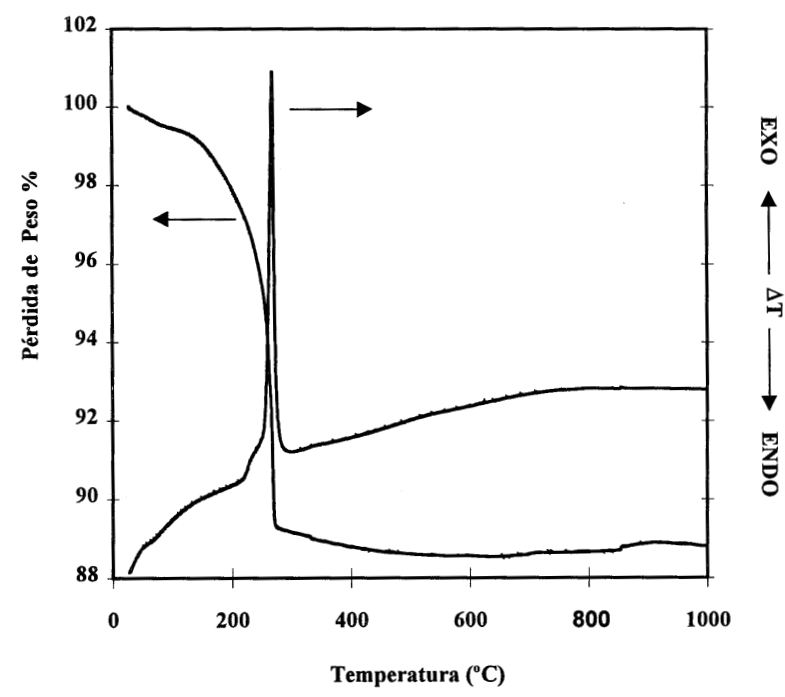

Figura 4.- Análisis termogravimétrico y térmico-diferencial del precipitado obtenido de la reacción del molibdato amónico y el nitrato hidratado de zinc con la temperatura. 


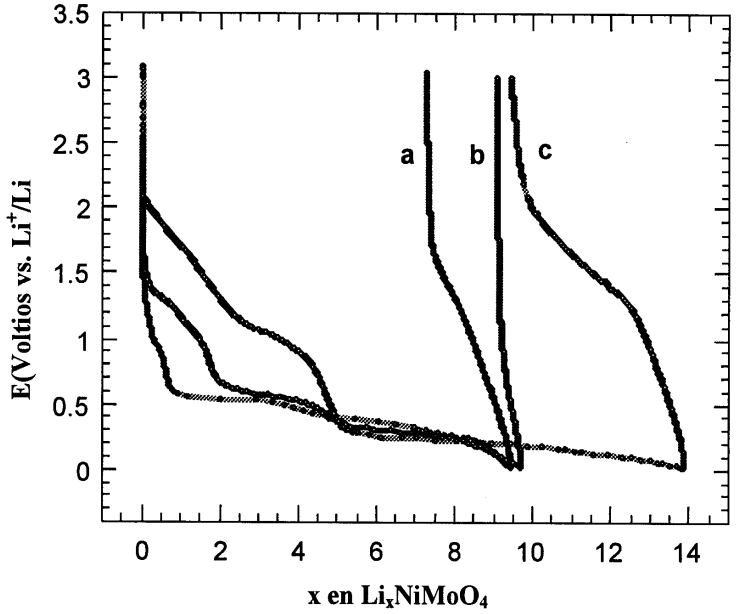

Figura 5.- Evolución del voltaje con la composición para celdas de configuración $\mathrm{Li} / \mathrm{NiMoO} 4$ descargadas hasta $0,02 \mathrm{~V}$ usando la fase preparada por reacción en estado sólido (a), reacción en solución calcinada a $900^{\circ} \mathrm{C}(\mathrm{b})$ y reacción en solución calciandada a $400^{\circ} \mathrm{C}$ (c).

embargo si se observaron diferencias apreciables en la cantidad de átomos de litio incorporados y en la forma de las curvas de voltaje frente a composición para las muestras preparadas por reacción en solución y calcinadas a diferente temperatura, es decir, mostrando diferente grado de cristalinidad. En general, los precipitados calcinados a menos temperatura incorporaron una mayor cantidad de átomos de litio por fórmula lo que probablemente se pueda relacionar con una menor cristalinidad y por tanto, posible presencia de un mayor número de posiciones disponibles para el ion huésped. Por ejemplo, en el caso del $\mathrm{NiMoO}_{4}$, el producto calcinado a $400^{\circ} \mathrm{C}$ y tal y como se muestra en la Fig.5 llega a incorporar hasta 14 átomos de litio por fórmula, un $40 \%$ más que el mismo producto calcinado a $900^{\circ} \mathrm{C}$ o que el sintetizado por RES. Este importante incremento en el número de átomos de litio incorporados en el precipitado calcinado a $400^{\circ} \mathrm{C}$ no puede ser debido solamente a inserción en el carbón o reducción del electrolito no siendo posible en este momento, explicar estas diferencias. Este efecto también se observó en el $\mathrm{ZnMoO}_{4}$ aunque en este caso y tal como se explicó anteriormente quedaría por aclarar la composición real del producto obtenido por RS y calcinado a $400^{\circ} \mathrm{C}$ ya que podría existir $\mathrm{ZnO}$ también presente aunque no visible por rayos-X. En cualquier caso, el proceso de inserción de litio en el intervalo de potencial de 3 a $0,02 \mathrm{~V}$ vs. $\mathrm{Li}^{+} / \mathrm{Li}$ en todos estos compuestos es irreversible.

Se comprobó que molibdatos isoestructurales como los de cobalto y níquel presentaban comportamientos electroquímicos diferentes lo que sugiere que el segundo metal presente en este tipo de compuestos además del molibdeno, juega un papel activo en la reacción de inserción.

Se realizaron experimentos en un intervalo más reducido de potencial descargando celdas similares a las anteriores hasta $0,5 \mathrm{~V}$ vs. $\mathrm{Li}^{+} / \mathrm{Li}$. Se observó que aunque disminuyó el número de átomos de litio incorporados al final de la primera descarga, la reversibilidad de la inserción del litio en algunos de estos molibdatos mejoró sustancialmente en este nuevo intervalo de potencial. En particular, los molibdatos de níquel (Fig.6) y cobalto incorporaron reversiblemente 2 átomos de litio ( el $50 \%$ de los inicialmente incorporados hasta $0,5 \mathrm{~V}$ ).

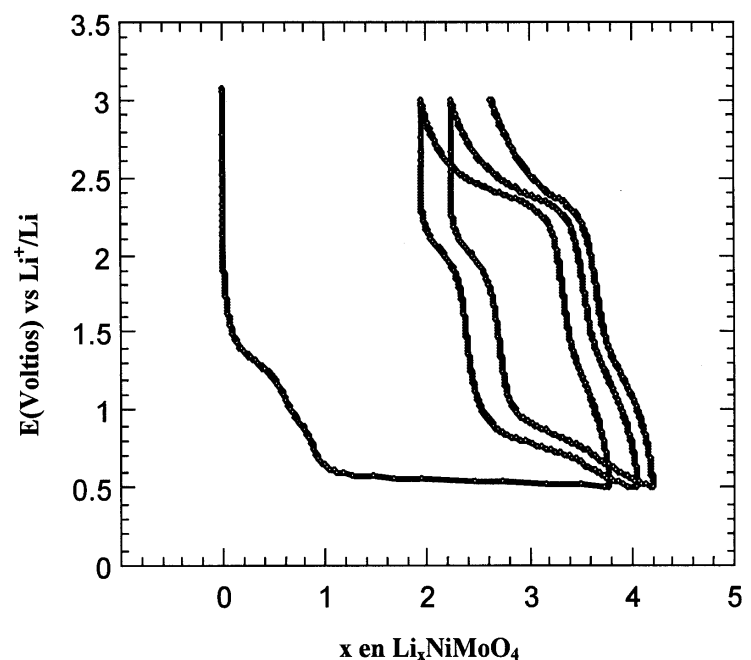

Figura 6.- Evolución del voltaje con la composición para una celda de configuración $\mathrm{Li} / \mathrm{NiMoO}_{4}$ descargada hasta 0,5V.

\section{CONCLUSIONES}

Se llevó a cabo un estudio de la inserción electroquímica de litio en varios molibdatos de aluminio y de metales divalentes preparados por dos métodos diferentes de síntesis: reacción de estado sólido y reacción en solución. No se observaron diferencias fundamentales en el comportamiento de los compuestos preparados por uno y otro método y calcinados a temperaturas elevadas aunque los productos preparados por reacción en solución y calcinados a temperaturas intermedias incorporaron en general una mayor cantidad de átomos de litio. Aunque la cantidad de litio incorporado cuando se descargaron las celdas hasta $0,02 \mathrm{~V}$ fue en general bastante elevada $(\mathrm{Li} / \Sigma \mathrm{M} \approx 4)$, la inserción de litio en ninguno de los compuestos estudiados mostró reversibilidad por lo que no serían buenos candidatos como electrodos en baterías recargables. Sin embargo en celdas descargadas hasta $0,5 \mathrm{~V}$ se observó reversiblidad en la inserción de 2 átomos de litio en $\mathrm{NiMoO}_{4}$ y $\mathrm{CoMoO}_{4}$.

\section{AGRADECIMIENTOS}

Los autores agradecen a CONACYT (Ref. 3862P-A9607) y a la UANL (PAICYT CA060-97) el apoyo financiero que permitió la realización de este trabajo.

\section{BIBLIOGRAFÍA}

1. Y. Idota, Eur. Pat. 0567149 A1 (1993).

2. Denis S, Baudrin E, Touboul M, Tarascon J-M, “Synthesis and Electrochemical 3. Properties of Amorphous Vanadates of General Formula $\mathrm{RVO}_{4}(\mathrm{R}=\mathrm{In}, \mathrm{Cr}$, Fe, Al Y) vs. Li", J. Electrochem. Soc. 144(12), 1997, 4099-4109.

3. Leroux F., Goward GR, Power WP and Nazar LF, “Understanding the Nature of Low-Potential Li Uptake into High Volumetric Capacity Molybdenum Oxides" Electrochemical Solid State Letters 1(6), 1998, 255-258.

4. Roberto Herrera Sanchez; Leopoldo Treviño; Antonio F. Fuentes; A. Martínez de la Cruz and Leticia M. Torres-Martínez, "Electrochemical Lithium Insertion in two Polymorphs of a Reduced Molybdenum Oxide $\left(\gamma\right.$ and $\gamma^{\prime}-\mathrm{Mo}_{4} \mathrm{O}_{11}{ }^{\prime \prime}, \mathrm{J}$. Solid State Electrochem. En prensa.

5. Mouget C, Chabre Y "Multichannel Potentiostatic and Galvanostatic System MacPile", Licensed from CNRS and UJF Grenoble to Bio-Logic Corp., 1 Av. De 1’Europe, F-38640, Claix, Francia. 